\title{
Correction to: Long-Term Oral Toxicity and Anti-osteoporotic Effect of Sintered Dicalcium Pyrophosphate in Rat Model of Postmenopausal Osteoporosis
}

\author{
Yuh-Feng Tsai ${ }^{1,2} \cdot$ Li-Ho Hsu $^{3,4} \cdot$ Chang-Chin $\mathrm{Wu}^{3,4,5} \cdot$ Wei-Hua Cai $^{6} \cdot \mathrm{Kai}^{-C h i a n g ~ \text { Yang }^{6}} \cdot$ Fang-Yu Fan $^{6}$ \\ Published online: 2 June 2018 \\ (c) Taiwanese Society of Biomedical Engineering 2018
}

\section{Correction to: J. Med. Biol. Eng. (2017) 37:181-190 https://doi.org/10.1007/s40846-016-0197-x}

The article "Long-Term Oral Toxicity and Anti-osteoporotic Effect of Sintered Dicalcium Pyrophosphate in Rat Model of Postmenopausal Osteoporosis", written by Yuh-Feng Tsai, Li-Ho Hsu, Chang-Chin Wu, Wei-Hua Cai, Kai-Chiang Yang and Fang-Yu Fan was originally published Online First without open access. After publication in volume [37], issue [2], page [181-190] the author decided to opt for Open Choice and to make the article an open access publication. Therefore, the copyright of the article has been changed to (C) The Author(s) [2018] and the article is forthwith distributed under the terms of the Creative Commons Attribution 4.0 International License (http://creativecommons.org/licen ses/by/4.0/), which permits use, duplication, adaptation,

The original article can be found online at https://doi.org/10.1007/ s40846-016-0197-x.

\section{Kai-Chiang Yang}

pumpkin@tmu.edu.tw

$\triangle$ Fang-Yu Fan

fish884027@tmu.edu.tw

1 Department of Diagnostic Radiology, Shin-Kong Wu Ho-Su Memorial Hospital, Taipei City 111, Taiwan

2 School of Medicine, Fu-Jen Catholic University, New Taipei City 24205, Taiwan

3 Department of Orthopedics, En Chu Kong Hospital, New Taipei City 23702, Taiwan

4 Department of Orthopedics, College of Medicine, National Taiwan University Hospital, National Taiwan University, Taipei City 10002, Taiwan

5 Department of Biomedical Engineering, Yuanpei University of Medical Technology, Hsinchu 30015, Taiwan

6 School of Dental Technology, College of Oral Medicine, Taipei Medical University, $250 \mathrm{Wu}$-Hsing Street, Taipei City 11031, Taiwan distribution and reproduction in any medium or format, as long as you give appropriate credit to the original author(s) and the source, provide a link to the Creative Commons license, and indicate if changes were made. 